\title{
Electron detachment from negative ions in bichromatic laser field
}

\author{
M. Yu. Kuchievł and V. N. Ostrovsky§ \\ School of Physics, University of New South Wales, Sydney 2052, Australia
}

\begin{abstract}
.
Negative ion detachment in two-colour laser field is considered within the recent modification of Keldysh model which makes it quantitatively reliable. The general approach is illustrated by calculation of angular differential detachment rates, partial rates for particular ATD (Above Threshold Detachment) channels and total detachment rates for $\mathrm{H}^{-}$ion in bichromatic field with 1:2 frequency ratio. Both perturbative and strong field regimes are examined. Polar asymmetry and phase effects are quantitatively characterized with some new features revealed. Phase effects are found to result in a huge anisotropy factor $\sim 10^{3}$ in the electron angular distribution in the perturbative regime.
\end{abstract}

\section{Introduction}

Photoionization of atoms in bichromatic laser field had received recently a considerable attention both in theory (see, for instance, Baranova et al 1990, 1992, 1993, Baranova and Zel'dovich 1991, Szöke et al 1991, Anderson et al 1992, Schafer and Kulander 1992, Potvliege and Smith 1991, 1992a,b, 1994, Pazdzersky and Yurovsky 1994, Protopapas et al 1994, Véniard et al 1995, Pazdzersky and Usachenko 1997, Fifirig et al 1997) and experiment (Muller et al 1990, Ce Chen and Elliott 1990, Baranova et al 1991, 1992, Yin et al 1992). One of the principal points of interest seems to be dependence of the observables on the difference of field phases $\varphi$, i.e. the problem of phase control. Another important aspect is the angular (polar) asymmetry of photoionization rate. These effects are interrelated since polar asymmetry is $\varphi$-dependent and vanishes for some particular value of phase (see more detail below). The calculations have been carried out previously for ionization of hydrogen atom in two laser fields with a frequency ratio 1:2 (Schafer and Kulander 1992), 1:3 (Potvliege and Smith 1991) and 2:3 (Potvliege and Smith 1994).

† E-mail: kuchiev@newt.phys.unsw.edu.au

$\S$ Permanent address: Institute of Physics, The University of St Petersburg, 198904 St.Petersburg, Russia; E-mail: Valentin.Ostrovsky@pobox.spbu.ru 
Potvliege and Smith (1992) presented results for various frequency ratios and initial states. Different schemes were employed, but all of them implied numerically intensive work.

For the multiphoton electron detachment from negative ions some analytical treatment exists (Baranova et al 1993, Pazdzersky and Yurovsky 1994, Pazdzersky and Usachenko 1997) being limited mostly to the case when one or both fields are weak. The presence of large number of parameters in the problem sometimes makes results of analytical studies not directly transparent. The case of fields with comparable (and large) intensities is also of interest bearing in mind both possible experimental realizations and the theoretical description of the transition between the multiphoton and tunneling regimes.

The multiphoton electron detachment from negative ions presents unique situation when quantitatively reliable results can be obtained by analytical methods in a broad range of parameters characteristic to the problem. Indeed, it has been demonstrated recently (Gribakin and Kuchiev 1997a,b) that judicious modification of the Keldysh (1964) model $\dagger$ ensures a very good quantitative agreement with results of numerically intensive developments, being much more simple. In many cases it also provides good agreement with available experimental data. It works unexpectedly well even for small number $n$ of photons absorbed. In addition to numerous examples considered previously (Gribakin and Kuchiev 1997a,b), here we briefly comment on the most recent experiments by Zhao et al (1997) on non-resonant excess photon detachment of negative hydrogen ions. After absorption of two photons, the electron is ejected in superposition of $S$ and $D$ waves due to selection rules. The experiment demonstrates prevalence of $D$ wave contribution $(90 \% \pm 10 \%)$. Our calculations give for $D$ and $S$ waves population $86.2 \%$ and $13.6 \%$ respectively $\ddagger$ for experimental conditions (light frequency $\omega=0.0428$, field intensity $I=10^{10} \mathrm{~W} / \mathrm{cm}^{2}$. The elaborate numerical calculations by Telnov and Chu (1996) and by Nikolopoulos and Lambropoulos (1997) give for $D$ wave population $91 \%$ and $89 \%$ respectively. The difference between these values is almost the same as the difference between our result and that of Nikolopoulos and Lambropoulos (1997), all three theoretical predictions lying within experimental error bars. This, together with the cases considered earlier allows us to suggest that even for $n=2$ an approach (Gribakin and Kuchiev 1997a,b) provides an accuracy comparable with that of the most elaborate numerical developments.

The present paper extends approach of Gribakin and Kuchiev (1997a,b) to the

$\dagger$ Subsequent development of this model was due to Perelomov et al (1966), Faisal (1973) and Reiss (1980); Perelomov and Popov (1967) were the first to consider multicolour process within this framework in terms of influence of higher harmonics on ionization probabilities.

‡ The model shows also some population of $G$ and higher partial waves. However, this unphysical effect proves to be less than $0.2 \%$ thus sustaining the model applicability. 
case of bichromatic field. Its objective is to provide the scheme and some representative quantitative results for two-colour electron detachment from negative ions. In particular, the phase effects and the polar asymmetry are studied. The number of parameters in the problem is quite large and at the moment they cannot be fixed experimentally. Nevertheless it seems to be worthwhile to carry out some pivoting calculations in order to obtain insight into the possible magnitude of effects specific for negative ions in bichromatic fields. We consider angular differential detachment rates, heights of ATD (Above Threshold Detachment) peaks and total detachment rates.

\section{Scheme of calculations}

The previously developed scheme (Gribakin and Kuchiev 1997a,b) needs some modifications to incorporate bichromatic problem when electric field in the light wave

$$
\vec{F}(t)=\vec{F}_{1} \cos \omega_{1} t+\vec{F}_{2} \cos \left(\omega_{2} t+\varphi\right)
$$

is a superposition of two harmonic components with the frequencies $\omega_{1}, \omega_{2}$ and the

amplitude vectors $\vec{F}_{1}, \vec{F}_{2}$ respectively; $\varphi$ is the difference of field phases. Atomic units are used throughout the paper unless stated otherwise.

We consider a case of commensurable field frequenciest which implies that the common period $T$ exists such that

$$
T=\frac{2 \pi}{\omega_{1}} M_{1}=\frac{2 \pi}{\omega_{2}} M_{2}
$$

for some mutually simple integers $M_{1}$ and $M_{2}$.

The exact expression for the differential transition rate $d w_{\lambda}$ is derived following Appendix A of the paper by Gribakin and Kuchiev (1997a) with the result

$$
\begin{aligned}
& d w_{\lambda}=2 \pi \sum_{\epsilon_{f}}\left|A_{\lambda \epsilon_{f}}\right|^{2} \delta\left(E_{\lambda}-E_{0}-\epsilon_{f}\right) \rho_{\lambda}, \\
& A_{\lambda \epsilon_{f}}=\frac{1}{T} \int_{0}^{T}\left\langle\psi_{\lambda}(t)|V(t)| \psi_{0}(t)\right\rangle d t .
\end{aligned}
$$

Here $\psi_{0}(t)=\exp \left(-i E_{0} t\right) \phi_{0}$ describes an initial state for the time-independent Hamiltonian $H_{0}$, and $\psi_{\lambda}(t)$ is a quasienergy state for the total Hamiltonian $H=$ $H_{0}+V(t)$, which includes interaction with the periodic field $V(t)=-e \vec{r} \cdot \vec{F}(t)$, $V(t)=V(t+T)$ :

$$
\begin{aligned}
i \frac{\partial \psi_{\lambda}}{\partial t} & =\left[H_{0}+V(t)\right] \psi_{\lambda}, \\
\psi_{\lambda}(t) & =\exp \left(-i E_{\lambda} t\right) \phi_{\lambda}, \quad \phi_{\lambda}(t)=\phi_{\lambda}(t+T),
\end{aligned}
$$

$\dagger$ The general treatment of incommensurable frequencies case was considered by Ho et al (1983), Delone et al (1984), Ho and Chu (1984), Manakov et al (1986), Potvliege and Smith (1992). 
$E_{\lambda}$ is the quasienergy, $\rho_{\lambda}$ is the density of states, $\vec{r}$ is the active electron vector. The energy $\epsilon_{f}$ absorbed by electromagnetic field could be presented as $\epsilon_{f}=n_{1} \omega_{1}+n_{2} \omega_{2}$ with some integers $n_{1}$ and $n_{2}$. However this representation (i.e. the choice of $n_{1}$ and $n_{2}$ ) generally is non-unique which reflexes existence of different coherent interfering paths (with different number of absorbed and emitted photons of each frequency) leading to the same final state.

If interaction of light wave with an electron is described in the dipole-length form, as presumed above, then a long range contribution to the matrix elements is emphasized. Therefore it is sufficient to employ an asymptotic form of the initial-state wave function (Gribakin and Kuchiev 1997a):

$$
\phi_{0}(\vec{r}) \approx A r^{\nu-1} \exp (-\kappa r) Y_{l m}(\hat{\vec{r}}) \quad(r \gg 1)
$$

where $E_{0}=-\frac{1}{2} \kappa^{2}, \nu=Z / \kappa, Z$ is the charge of the atomic residual core $(\nu=Z=0$ for a negative ion), and $\hat{\vec{r}}$ is the unit vector. The coefficients $A$ are tabulated for many negative ions (Radzig and Smirnov 1985).

The amplitude $A_{\lambda \epsilon_{f}}(4)$ is evaluated neglecting the influence of atomic potential on the photoelectron in the final state. Further on, the integral over time in (4) is calculated within the stationary phase approximation which presumes large magnitude of the classical action

$$
S(t)=\frac{1}{2} \int^{t}\left(\vec{p}+\vec{k}_{t^{\prime}}\right)^{2} d t^{\prime}-E_{0} t
$$

where $\vec{k}_{t}$ is the classical electron momentum due to the field

$$
\vec{k}_{t}=e \int^{t} \vec{F}\left(t^{\prime}\right) d t^{\prime}
$$

The photoelectron translational momentum $\vec{p}$ plays a role of the quantum number $\lambda$ above; in particular, the quasienergy $E_{\lambda}=E_{\vec{p}}$,

$$
E_{\vec{p}}=\frac{1}{2} \vec{p}^{2}+\frac{e^{2}}{4 \omega_{1}^{2}} F_{1}^{2}+\frac{e^{2}}{4 \omega_{2}^{2}} F_{2}^{2}
$$

includes contribution from the electron quiver energy due to the field.

The result of calculations of the amplitude (4) could be written as a modification of the formula (25) in the paper by Gribakin and Kuchiev (1997a):

$$
A_{\vec{p} \epsilon_{f}}=-\frac{(2 \pi)^{2}}{T} A \Gamma(1+\nu / 2) 2^{\nu / 2} \kappa^{\nu} \sum_{\mu} Y_{l m}\left(\hat{\vec{p}}_{\mu}\right) \frac{\exp \left(i S_{\mu}\right)}{\sqrt{2 \pi\left(-i S_{\mu}^{\prime \prime}\right)^{\nu+1}}} .
$$

A corresponding expression for the detachment rate for the negative ion case $(\nu=0)$ reads:

$$
\frac{d w_{e_{f}}}{d \Omega_{\vec{p}}}=\frac{1}{(2 \pi)^{2}} p\left|A_{\vec{p} \epsilon_{f}}\right|^{2}=\frac{(2 \pi)^{2}}{T^{2}} p A^{2}\left|\sum_{\mu} Y_{l m}\left(\hat{\vec{p}}_{\mu}\right) \frac{\exp \left(i S_{\mu}\right)}{\sqrt{2 \pi S_{\mu}^{\prime \prime}}}\right|^{2}
$$


Here the subscript $\mu$ indicates that the function is calculated at the saddle point $t=t_{\mu}$ which satisfies equation

$$
S^{\prime}\left(t_{\mu}\right)=0
$$

In the plane of the complex-valued time the saddle points $t_{\mu}$ lie symmetrically with respect to the real axis. Summation in (11) includes the points lying in the upper half plane $\left(\operatorname{Im} t_{\mu}>0\right)$ with $0 \leq \operatorname{Re} t_{\mu} \leq T$; $\hat{\vec{p}}_{\mu}$ is a unit vector in the direction of $\vec{p}+\vec{k}_{\mu}$. The magnitude of the final electron translational momentum $p$ is governed by the energy conservation

$$
\frac{1}{2} \kappa^{2}+E_{\vec{p}}=\epsilon_{f}
$$

which ensures that the momentum is real for open ATD channels.

According to (8), (9), (1) we have

$$
\begin{aligned}
& S^{\prime}(t)=\frac{1}{2}\left(\vec{p}+\vec{k}_{t}\right)^{2}+\frac{1}{2} \kappa^{2}= \\
& =\frac{1}{2} \vec{p}^{2}+\frac{e^{2}}{2 \omega_{1}^{2}} F_{1}^{2} \sin ^{2} \omega_{1} t+\frac{e^{2}}{2 \omega_{2}^{2}} F_{2}^{2} \sin ^{2}\left(\omega_{2} t+\varphi\right)+ \\
& +p \cdot F_{1} \frac{e}{\omega_{1}} \sin \omega_{1} t+p \cdot F_{2} \frac{e}{\omega_{2}} \sin \left(\omega_{2} t+\varphi\right)+ \\
& +\vec{F}_{1} \cdot \vec{F}_{2} \frac{e^{2}}{\omega_{1} \omega_{2}} \sin \omega_{1} t \sin \left(\omega_{2} t+\varphi\right)+\frac{1}{2} \kappa^{2} .
\end{aligned}
$$

The frequencies $\omega_{1}$ and $\omega_{2}$ are integer multiples of the basic frequency $\omega=2 \pi / T$

$$
\omega_{1}=M_{1} \omega, \quad \omega_{2}=M_{2} \omega .
$$

Assuming for definiteness that $M_{2}>M_{1}$ and introducing $\zeta=\exp (i \omega t)$ we notice that the function

$$
\mathcal{P}(\zeta)=\zeta^{2 M_{2}} S^{\prime}(\zeta)
$$

is a polynomial of the power $4 M_{2}$ in $\zeta$. This observation is of practical importance since equation (13) defining the saddle point is equivalent to

$$
\mathcal{P}(\zeta)=0 .
$$

The efficient numerical procedures for finding roots of polynomials are available, and, in particular, one can be confident that all the roots are found.

The practical calculations are conveniently carried out using the Mathematica program (Wolfram 1991). Starting from the expression for $S^{\prime}(t)$ one derives $S(t)$ and $S^{\prime \prime}(t)$ by analytical integration and differentiation respectively. The saddle points are found using Eq.(18), and the roots $t_{\mu}$ lying in the upper half plane are selected. Finite summation over $\mu$ in (11) or (12) completes the calculation. 
The roots $t_{\mu}$ and hence the photoionization amplitude (11) and rate (12) depend on the orientation of electron translational momentum $\vec{p}$ with respect to the field amplitudes $\vec{F}_{1}$ and $\vec{F}_{2}$. It is not difficult to consider the fields of various relative orientation and polarization, but for simplicity we limit our further calculations to linear polarized fields with $\vec{F}_{1} \| \vec{F}_{2}$. Then the differential photoionization rate depends only on the single angle $\theta$ between the vectors $\vec{p}$ and $\vec{F}_{1} \| \vec{F}_{2}$.

\section{Results}

Our calculations for $\mathrm{H}^{-}$detachment are carried out for the parameters $\kappa=0.2354$, $A=0.75$ (Radzig and Smirnov 1985). The frequencies ratio $\omega_{1} / \omega_{2}=1: 2$ is considered. In this case the field (1) have zero mean value, but possesses polar asymmetry (i.e. asymmetry under inversion of the $z$ axis directed along $\vec{F}_{1} \| \vec{F}_{2}$ ) which could be conveniently characterized by the time-average value (Baranova et al 1990)

$$
\left\langle F^{3}\right\rangle=\frac{3}{4} F_{1}^{2} F_{2} \cos \varphi
$$

Presuming that $F_{1}, F_{2}>0$, from this expression one infers, for instance, that for the phase $\varphi \in\left[0, \frac{1}{2} \pi\right]$ the electric field effectively attains larger values in the positive$z$ direction than in the negative- $z$ one. This is illustrated, for example, by Fig. 1 in the paper by Schafer and Kulander (1992), or by Fig.2 in the paper by Baranova et al (1993). Note that our definition of the phase $\varphi$ is the same as in the papers by Baranova et al (1990), Muller et al (1990), Pazdzersky and Yurovsky (1995), but differs from that chosen by Schafer and Kulander (1992) who describe the electric filed as $\vec{F}(t)=\vec{F}_{1} \sin \omega_{1} t+\vec{F}_{2} \sin \left(\omega_{2} t+\varphi_{\mathrm{KS}}\right)$. Namely, the phases are related as $\varphi_{\mathrm{KS}}=\varphi-\frac{1}{2} \pi$.

Although the formula (19) shows that the field has polar symmetry for $\varphi=\frac{1}{2} \pi$ and the maximal polar asymmetry for $\varphi=0$, quite paradoxically, the differential detachment rate (12) possesses polar symmetry for $\varphi=0$ (i.e. is invariant under transformation $\theta \Rightarrow \pi-\theta$ ), and is asymmetrical for other values of phase (see discussion by Baranova et al (1990), Schafer and Kulander (1992), Pazdzersky and Yurovsky (1995)).

The other features of the phase effects are as follows.

(i) The system Hamiltonian is a $2 \pi$-periodic function of the parameter $\varphi$.

(ii) The system Hamiltonian is not changed by simultaneous transformation $\varphi \Rightarrow-\varphi$, $\theta \Rightarrow \pi-\theta$. The same applies to the differential detachment rate (12).

(iii) The transformation $\varphi \Rightarrow \pi-\varphi$ leaves the Hamiltonian invariant only if $t$ is replaced by $-t$.

As stressed by Baranova et al (1990), Baranova and Zeldovich (1991), Anderson et al (1992), Baranova et al (1993), the problem is invariant under the time inversion 
operation provided the final-state electron interaction with the atomic core is neglected. This is the case in the present model. Therefore our differential ionization rates are the same for $\varphi$ and $(\pi-\varphi)$; hence it is sufficient for us to consider phases only from the interval $\varphi \in\left[0, \frac{1}{2} \pi\right]$. The calculations by Baranova et al (1990) within the perturbation theory and by Schafer and Kulander (1992) within the wave propagation technique took into account the final state electron-core interaction. Therefore they have found some deviations from the symmetry under $\varphi \Rightarrow(\pi-\varphi)$ transformation. However, for the negative ion photodetachment this effect could be anticipated to have only minor influence.

At first we consider two fields with the frequencies $\omega=0.0043$ and $2 \omega$ and equal intensities $I_{1}=I_{2}=10^{9} \mathrm{~W} / \mathrm{cm}^{2}$. It is well known that the regime of detachment process is governed by the Keldysh parameter $\gamma=\omega \kappa / F(\gamma \gg 1$ for multiphoton detachment in perturbative regime; $\gamma \ll 1$ for strong field, or tunneling regime). In the present case for the first field we have $\gamma_{1}=\omega_{1} \kappa / F_{1}=6$, and for the second field $\gamma_{2}=2 \gamma_{1}$, which corresponds to multiphoton regime.

Fig.1 (as well as Figs.2-4 below) shows differential detachment rate as a function of the angle $\theta$. The abscissas of the plots give the magnitude of

$$
\frac{1}{2} \frac{1}{2 \pi} \frac{d w_{e_{f}}}{d \cos \theta}=\frac{1}{(2 \pi)^{2}} p\left|A_{\vec{p} \epsilon_{f}}\right|^{2},
$$

where the right hand side was calculated using the right hand side of the formula (12). The left hand side of Eq.(20) has the factor $\frac{1}{2}$. It means that the true detachment rate in case of $\mathrm{H}^{-}$ion is twice larger than that given by Eq.(12). By this we account for the two possible spin states of residual $\mathrm{H}$ atom (i.e. for the presence of two equivalent electrons in $\mathrm{H}^{-}$).

In Fig.1 we show the differential detachment rate for the first and second ATD peaks which correspond to absorption of $n=7$ and $n=8$ photons of frequency $\omega$ respectively. In Fig. 2 the same results are shown but for doubled value of the field amplitude $F_{2}$. In Fig.3 the amplitude $F_{2}$ is the same as in Fig.1, but the amplitude $F_{1}$ is doubled.

In all cases the angular distributions exhibit strong dependence on the field phase difference $\varphi$. This is well expected since the angular patterns are formed by interference of contributions coming from different complex-valued moments of time $t_{\mu}$. For $\varphi=0$ the distribution is rather flat, with $\varphi$ increasing it becomes more oscillatory. An interesting, and not obvious feature is that for $\varphi=\frac{1}{2} \pi$ the rate turns zero at the values of angle $\theta$ where it has minimum.

In Fig. 4 we present the results for the same frequencies as before and equal field intensities $I_{1}=I_{2}=10^{11} \mathrm{~W} / \mathrm{cm}^{2}$. Here the Keldysh parameter for the first field is $\gamma_{1}=\omega_{1} \kappa / F_{1}=0.6$. Bearing in mind the presence of the second field one can suppose that the situation corresponds to the onset of strong-field domain. The first open ATD channel corresponds to absorption of $n=18$ photons of frequency $\omega$. The patterns in 
differential rates become more oscillatory than in the weak filed case.

The partial detachment rate for each ATD channel is obtained by integration of (12) over angles

$$
w_{e_{f}}=\int \frac{d w_{e_{f}}}{d \Omega_{\vec{p}}} d \Omega_{\vec{p}}=\int_{\theta=\pi}^{\theta=0} \frac{d w_{e_{f}}}{d \cos \theta} d \cos \theta .
$$

We present separately the result $w_{e_{f}}^{(u)}$ of integration over the upper half-space of the electron ejection $\left(0<\theta<\frac{1}{2} \pi\right)$ and its counterpart $w_{e_{f}}^{(l)}$ for the lower half-space $\left(\frac{1}{2} \pi<\theta<\pi\right)$. These magnitudes give a bulk characterization for the partial rate polar asymmetry. As discussed above, the polar asymmetry disappears (i.e. $w_{N}^{(u)}=w_{N}^{(l)}$ ) for $\varphi=0$. In the perturbative regime (Fig.5) for the same conditions as in Fig.1 we see that the bulk polar asymmetry parameter $w_{N}^{(u)} / w_{N}^{(l)}$ exceeds $10^{3}$ even for the lowest ATD channel $(N=1)$ provided the phase $\varphi$ is not too small (the open ATD channels are labeled by the number $N=1,2, \ldots$ in order of increasing emitted electron energy). For higher ATD peaks the bulk asymmetry is even larger.

The partial detachment rates integrated over all ejection angles $w_{N}=w_{N}^{(u)}+w_{N}^{(l)}$ is shown in Fig. 6 for three representative values of $\varphi$. Even for $N=1$ the variation of the phase $\varphi$ leads to the substantial change in the detachment rate described by a factor 4 .

In the tunneling regime the bulk polar asymmetry (Fig.7) is not as prominent as in the perturbative regime. Nevertheless it is quite substantial. Note that both in Fig.5 and Fig. 7 the electron emission in the upper half-space $\left(0<\theta<\frac{1}{2} \pi\right)$ is more probable for all $N$, i.e. $w_{N}^{(u)}>w_{N}^{(l)}$ in the interval of phases considered $\left(\varphi \in\left[0, \frac{1}{2} \pi\right]\right)$. As discussed above (see the property (ii)), the situation is reversed for $\varphi \in\left[-\frac{1}{2} \pi, 0\right]$. For small value of the phase $\varphi=\frac{1}{8} \pi$ in Fig.7 there is a clear tendency to swap the relation between $w_{N}^{(u)}$ and $w_{N}^{(l)}$ for higher values of $N$ which is prevented by a kind of 'pseudocrossing'. For larger $\varphi$ the partial rates $w_{N}^{(l)}$ are strongly suppressed when $N$ increases. The phase effects in the partial rates $w_{N}$ are less significant in the tunneling regime (Fig.8).

The total detachment rates are obtained by summation over all open ATD channels:

$$
w=\sum_{N} w_{N}
$$

The results for the total rates as well as $w^{(l, u)}=\sum_{N} w_{N}^{(l, u)}$ are presented in table 1. In the perturbative regime we see again strong bulk asymmetry (three orders of magnitude and more) if the phase difference $\varphi$ is not close to zero, and substantial variation of $w$ with $\varphi$. Actually this result reiterates that for the partial rate with $N=1$ since the latter gives a dominant contribution to the total rate in the perturbative regime.

In the strong field regime the bulk polar asymmetry $w^{(l)} / w^{(u)}$ remains well manifested in the rate summed over all ATD channels. However, the total rate $w$ is practically insensitive to the phase variation. The partial rates $w_{N}$ in Fig. 8 exhibit some oscillatory structure as functions of $N$ with position of extrema depending on $\varphi$. 
This $\varphi$-dependence almost completely disappears after summation over $N$ as table 1 shows.

\section{Conclusion}

As a summary, the approach of Gribakin and Kuchiev (1997a,b) provides convenient tool for investigating two-colour photodetachment of negative ions. The bichromatic electron detachment for $\mathrm{H}^{-}$ion in the fields with 1:2 frequency ratio is examined in the perturbative and tunneling regimes. The polar asymmetry is found to be tremendously strong $\left(\sim 10^{3}\right)$ in the perturbative regime. Note that the asymmetry remains strong and keeps the same sign for all ATD for a wide range of phases $0<\varphi<\pi$. This property makes this effect be convenient for experimental observation because it manifests itself very strongly even for a relatively poor resolution of the phase $\delta \varphi \sim \pi / 2$. It should be noted that via the recoil mechanism the predicted effect leads also to acceleration of the core thus creating anisotropic flux of neutral $\mathrm{H}$ atoms.

\section{Acknowledgments}

We appreciate fruitful discussions with G.F.Gribakin. One of us (M.Yu.K.) is thankful to the Australian Research Council for support. This work was supported by the Australian Bilateral Science and Technology Collaboration Program. V.N.O. acknowledges a hospitality of the staff of the School of Physics of UNSW where this work was fulfilled.

\section{References}

Anderson D Z, Baranova N B, Green K, and Zel'dovich B Ya 1992 Zh. Eksp. Teor. Fiz. 102 397-405 [1992 Sov. Phys.-JETP 75 210-4]

Baranova N B, Beterov I M, Zel'dovich B Ya, Ryabtsev I I, Chudinov A N and Shul'ginov A A 1992 Pis'ma Zh. Eksp. Teor. Fiz. 55 431-5 [1992 JETP Letters 55 439-44]

Baranova N B, Zel'dovich B Ya, Chudinov A N and Shul'ginov A A 1990 Zh. Eksp. Teor. Fiz. 98 1857-68 [1990 Sov. Phys.-JETP 71 1043-9]

Baranova N B and Zel'dovich B Ya 1991 J. Opt. Soc. Am. B 8 27-32

Baranova N B, Reiss H R and Zel'dovich B Ya 1993 Phys. Rev. A 48 1497-505

Ce Chen and Elliott D S 1990 Phys. Rev. Lett. 65 1737-40

Delone N B, Manakov N L and Fainshtein A G 1984 Zh. Eksp. Teor. Fiz. 86 906-14 [1984 Sov. Phys.JETP 59 529-33]

Gribakin G F and Kuchiev M Yu 1997a Phys. Rev. A 55 3760-71

—1997b J. Phys. B: At. Mol. Opt. Phys. 30 L657-64

Faisal F H M 1973 J. Phys. B: At. Mol. Phys. 6 L89-92

Fifirig M, Cionga A and Florescu V 1997 J. Phys. B: At. Mol. Opt. Phys. 30 2599-608

Ho T S, Chu S I and Tietz J V 1983 Chem. Phys. Lett. 96 464-71

Ho T S and Chu S I 1984 J. Phys. B: At. Mol. Opt. Phys. 17 2101-28 
Keldysh L V 1964 Zh. Eksp. Teor. Fiz. 47 1945-57 [1965 Sov. Phys.-JETP 20 1307-14]

Nikolopoulos L A A and Lambropoulos P 1997 Phys. Rev. A 56 3106-15

Manakov N L, Ovsiannikov V D and Rapoport L P 1986 Phys.Rep. 141 319-433

Muller H G, Bucksbaum P H, Schumacher D W and Zavriev A 1990 J. Phys. B: At. Mol. Opt. Phys. $232761-9$

Pazdzersky V A and Yurovsky V A 1991 J. Phys. B: At. Mol. Opt. Phys. 24 733-40

-1994 Phys. Rev. A 51 632-40

Pazdzersky V A and Usachenko V I 1997 J. Phys. B: At. Mol. Opt. Phys. 30 3387-402

Perelomov A M, Popov V S and Terent'ev M V 1966 Zh. Eksp. Teor. Fiz. 50 1393-409 [1966 Sov. Phys.-JETP 23 924-34]

Perelomov A M and Popov V S 1967 Zh. Eksp. Teor. Fiz. 52 514-26 [1967 Sov. Phys.-JETP 25 336-43]

Potvliege R M and Smith P H G 1991 J. Phys. B: At. Mol. Opt. Phys. 24 L641-6

—1992 J. Phys. B: At. Mol. Opt. Phys. 25 2501-16

—1994 Phys. Rev. A 49 3110-3

Protopapas M, Knight P L and Burnett K 1994 Phys. Rev. A 49 1945-9

Radzig A A and Smirnov B M 1985 Reference Data on Atoms, Molecules and Ions (Berlin: Springer)

Reiss H R 1980 Phys. Rev. A 22 1786-813

Schafer K J and Kulander K C 1992 Phys. Rev. A 45 8026-33

Szöke A, Kulander K C and Bardsley J N 1991 J. Phys. B: At. Mol. Opt. Phys. 24 3165-71

Telnov D A and Chu S I 1996 J. Phys. B: At. Mol. Opt. Phys. 29 4401-10

Véniard V, Taleb R and Maquet A 1995 Phys. Rev. Lett. 74 4161-4

Wolfram S 1991 Mathematica: A System for Doing Mathematics by Computer, 2nd ed. (Addison-Wesley Publishing Co., Palo Alto)

Yin Y-Y, Ce Chen and Elliott D S 1992 Phys. Rev. Lett. 69 2353-6

Zhao X M, Gulley M S, Bryant H C, Strauss C E M, Funk D J, Stintz A, Rislove D C, Kyrala G A, Ingalls W B and Miller W A 1997 Phys. Rev. Lett. 78 1656-9 
Table 1. Total rates $w$ (summed over all ATD channels) for detachment of $\mathrm{H}^{-}$ion in the bichromatic field with the frequencies $\omega=0.0043$ and $2 \omega$, equal intensities $I_{1}=I_{2}$ and some representative values of the phase difference $\varphi$. The detachment rates $w^{(u)}$ and $w^{(l)}$ for electron ejection into the upper and lower half-spaces respectively are also shown.

\begin{tabular}{|c||l|l|l||l|l|l|}
\hline$\varphi$ & $w$ & $w^{(u)}$ & $w^{(l)}$ & $w$ & $w^{(u)}$ & $w^{(l)}$ \\
\hline & \multicolumn{2}{||}{ in units $10^{-21}$ a.u. } & \multicolumn{2}{||}{ in units $10^{-6}$ a.u. } \\
\hline \hline 0 & \multicolumn{1}{||}{1.55} & 0.773 & 0.773 & 162.1 & 81.1 & 81.1 \\
\hline$\frac{1}{8} \pi$ & 2.12 & 1.88 & 0.240 & 164.6 & 99.8 & 64.8 \\
\hline$\frac{1}{4} \pi$ & 3.56 & 3.50 & 0.00566 & 164.8 & 113.0 & 51.8 \\
\hline$\frac{3}{8} \pi$ & 5.07 & 5.06 & 0.00112 & 165.1 & 123.8 & 41.4 \\
\hline$\frac{1}{2} \pi$ & 5.71 & 5.71 & 0.00040 & 164.9 & 128.0 & 37.0 \\
\hline
\end{tabular}




\section{Figure captions}

Figure 1. Detachment of $\mathrm{H}^{-}$ion in bichromatic field with the frequencies $\omega=0.0043$ and $2 \omega$ and equal intensities $I_{1}=I_{2}=10^{9} \mathrm{~W} / \mathrm{cm}^{2}$. Differential detachment rate (see Eq.(20)) (in units $10^{-12}$ a.u.) as a function of the angle $\theta$ is shown for the first ( $a$, absorption of $n=7$ photons of frequency $\omega$ ) and second ( $b, n=8)$ ATD peaks and various values of the field phase difference: solid curve $-\varphi=0$; short-dashed curve

- $\varphi=\frac{1}{8} \pi$; dot-dashed curve $-\varphi=\frac{1}{4} \pi$; dotted curve $-\varphi=\frac{3}{8} \pi$; long-dashed curve $\varphi=\frac{1}{2} \pi$.

Figure 2. Same as in Fig.1, but for unequal field intensities $I_{1}=10^{9} \mathrm{~W} / \mathrm{cm}^{2}$, $I_{1}=4 \times 10^{9} \mathrm{~W} / \mathrm{cm}^{2}$. The differential detachment rates is shown in units $10^{-10}$ a.u.

Figure 3. Same as in Fig.1, but for unequal field intensities $I_{1}=4 \times 10^{9} \mathrm{~W} / \mathrm{cm}^{2}$, $I_{1}=10^{9} \mathrm{~W} / \mathrm{cm}^{2}$. The differential detachment rate is shown in units $10^{-10}$ a.u.

Figure 4. Same as in Fig.1, but in the strong field regime: $I_{1}=I_{2}=10^{11} \mathrm{~W} / \mathrm{cm}^{2}$. The detachment rate for the first ( $a$, absorption of $n=18$ photons of frequency $\omega$ ) and second $(b, n=19)$ ATD peaks is shown in units $10^{-6}$ a.u. 
Figure 5. Partial detachment rates for various ATD channels for $\mathrm{H}^{-}$ion in bichromatic field with the same parameters as in Fig.1 (perturbative regime) and various values of the field phase difference $\varphi . N$ labels ATD peaks with the lowest $N=1$ peak corresponding to absorption of 7 photons of frequency $\omega=0.0043$. The rate $w_{N}^{(u)}$ of electron emission in the upper half-space is shown by circles, its counterpart $w_{N}^{(l)}$ for the lower half-space is depicted by triangles. The plot for $\varphi=\frac{1}{8} \pi$ additionally includes the rate for $\varphi=0$ (crosses) when emission is polar symmetrical $\left(w_{N}^{(u)}=w_{N}^{(l)}\right)$. The symbols are joined by lines to help the eye.

Figure 6. Same as in Fig.5, but for the detachment rate integrated over all angles $w_{N}=w_{N}^{(u)}+w_{N}^{(l)}$. The results for three values of the phase $\varphi$ are shown: circles $\varphi=0 ;$ blocks $-\varphi=\frac{1}{4} \pi$, triangles $-\varphi=\frac{1}{2} \pi$.

Figure 7. Same as in Fig.5, but for the field parameters chosen as in Fig.4 (strong field regime). The lowest $N=1$ peak corresponds to absorption of 18 photons of frequency $\omega=0.0043$. The detachment rate is shown in units $10^{-6}$ a.u.

Figure 8. Same as in Fig.6, but for the field parameters chosen as in Fig.7 (strong field regime). The detachment rate is shown in units $10^{-6}$ a.u. 


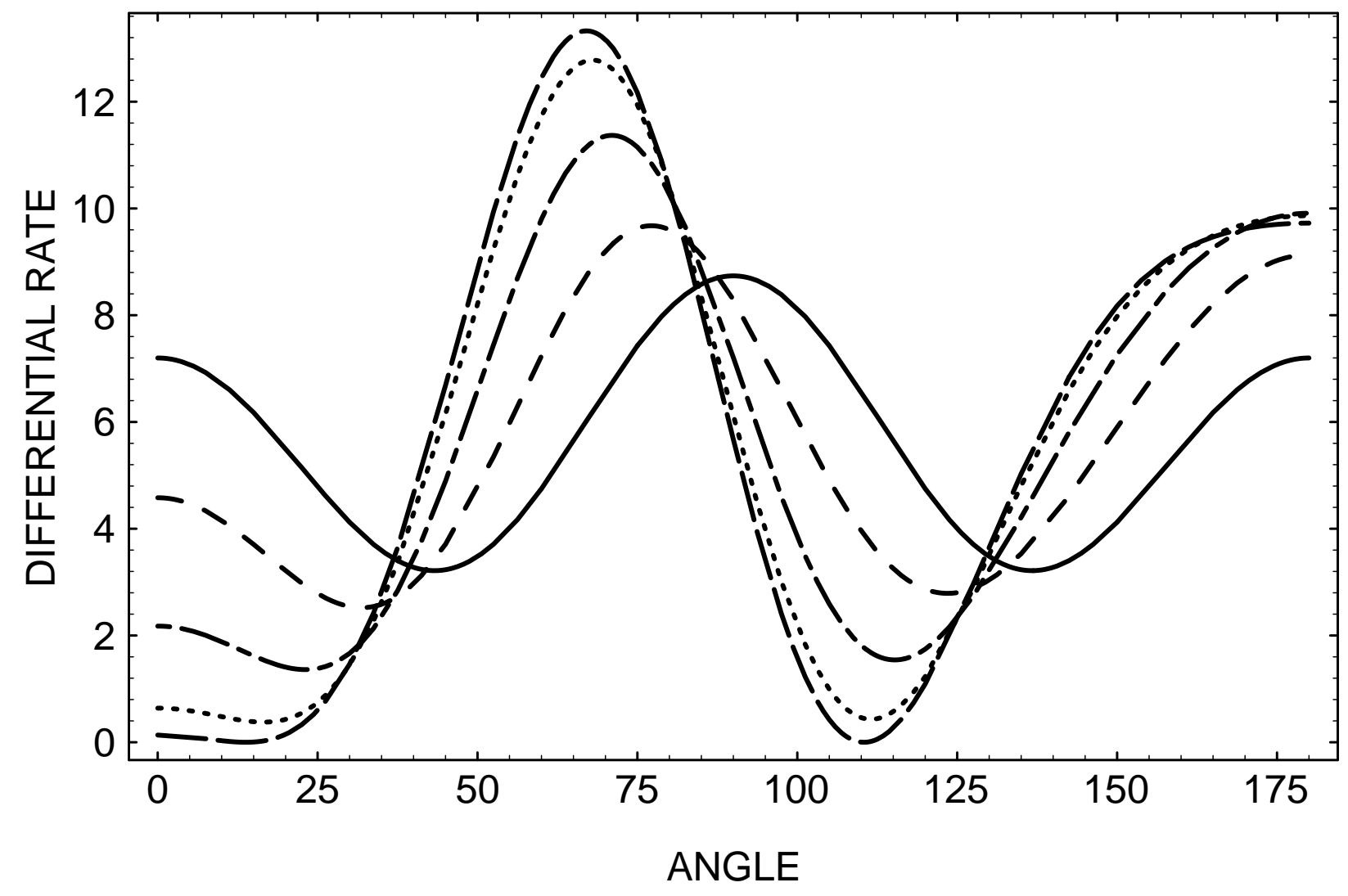




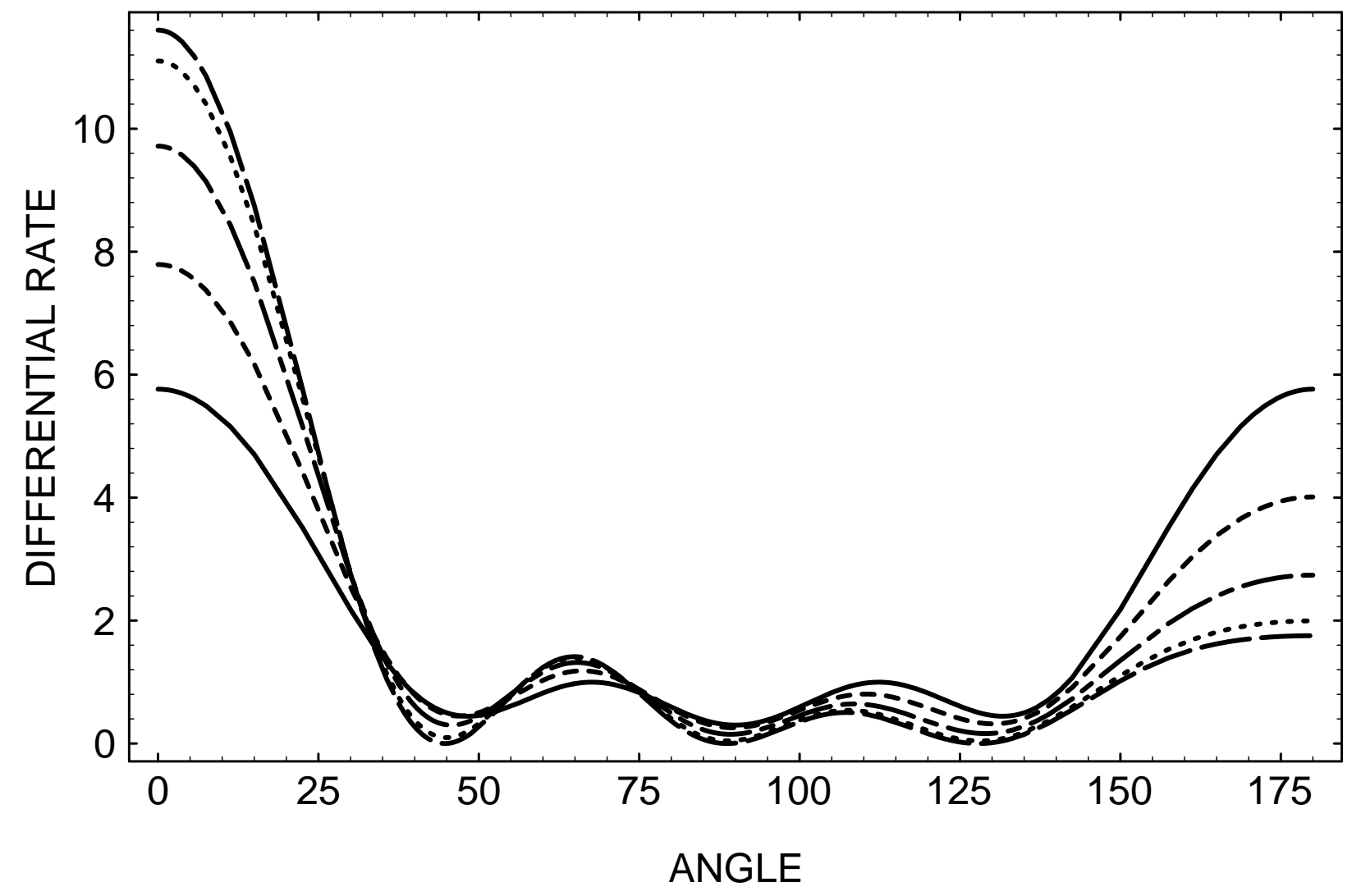




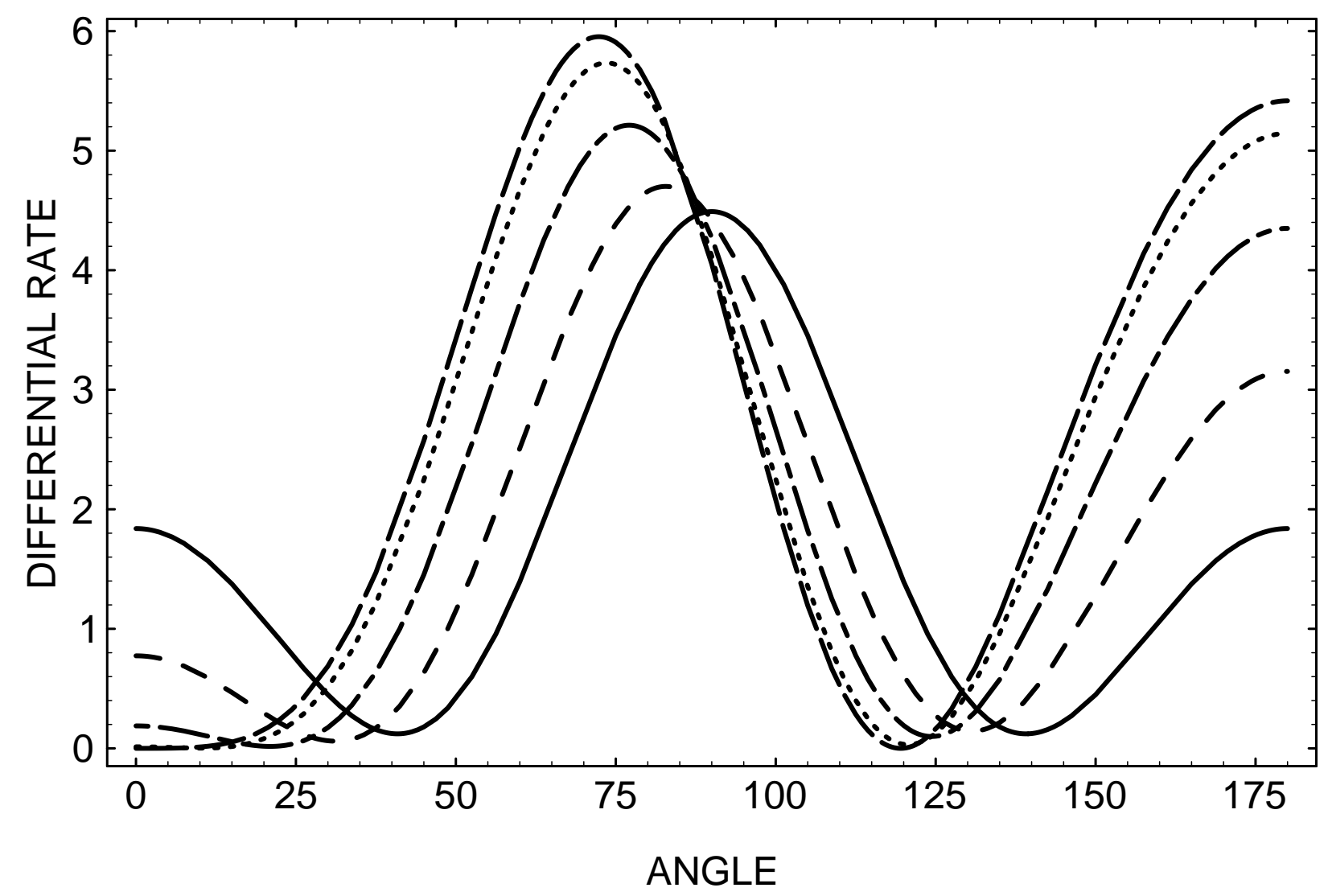




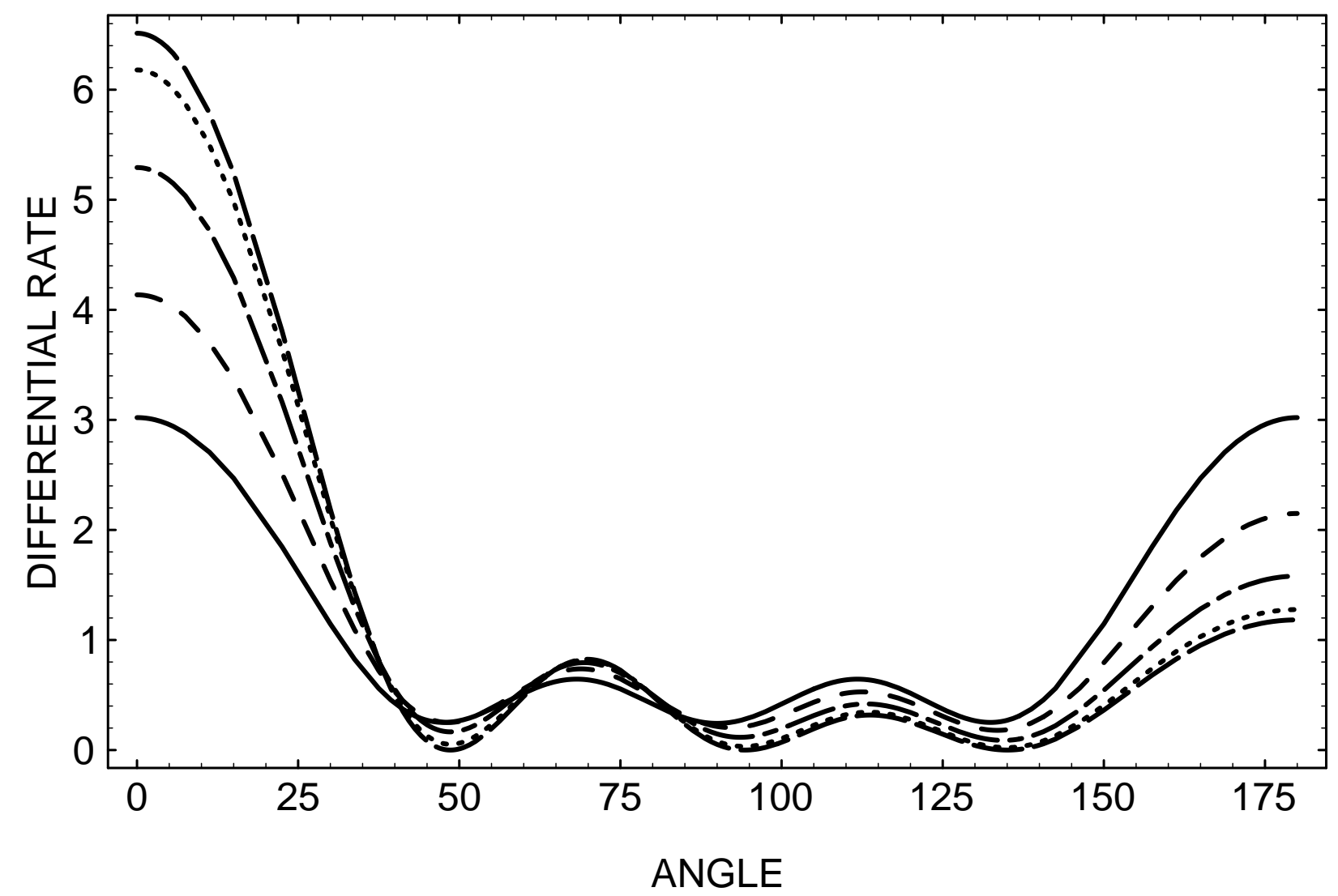




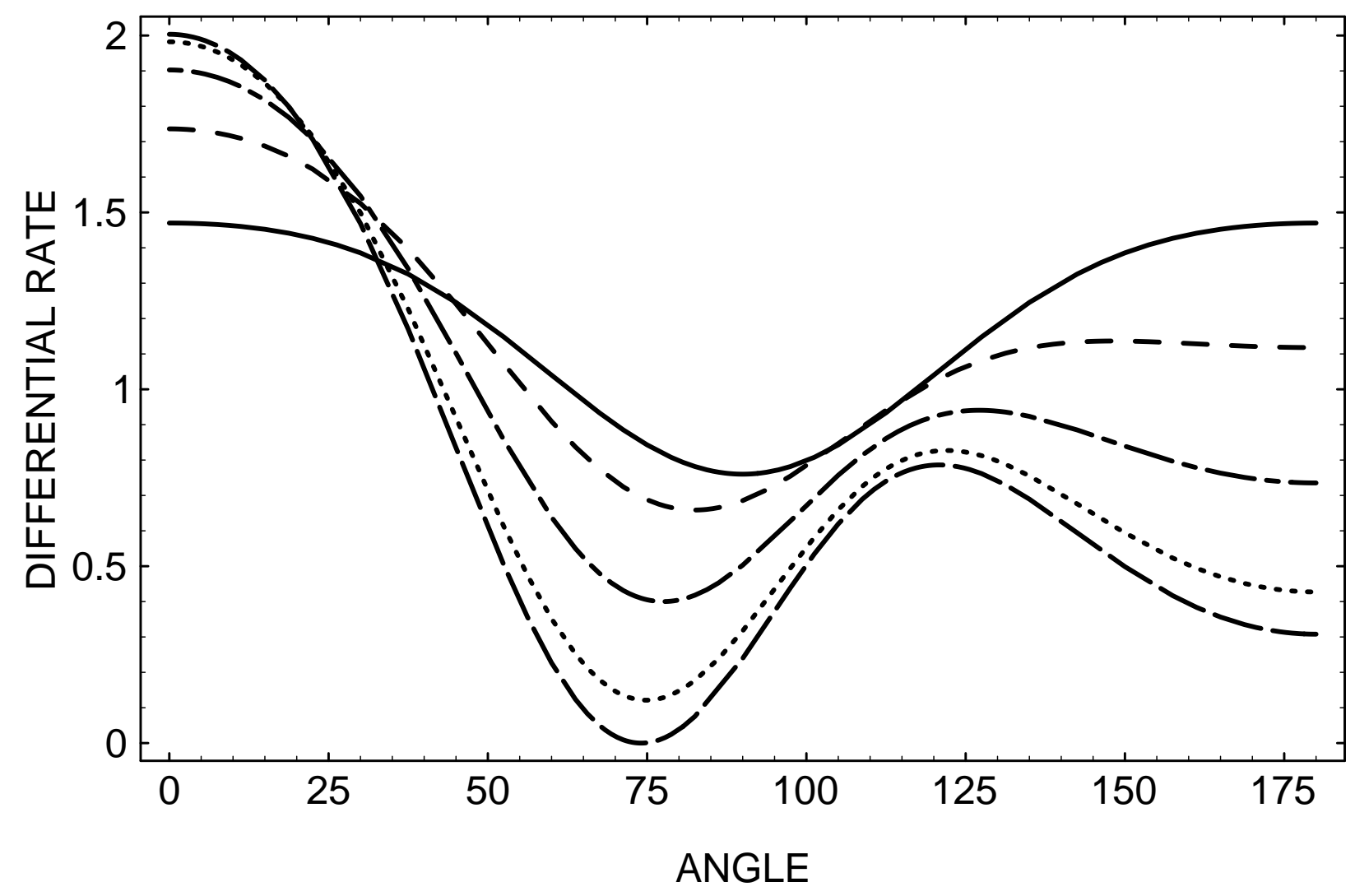




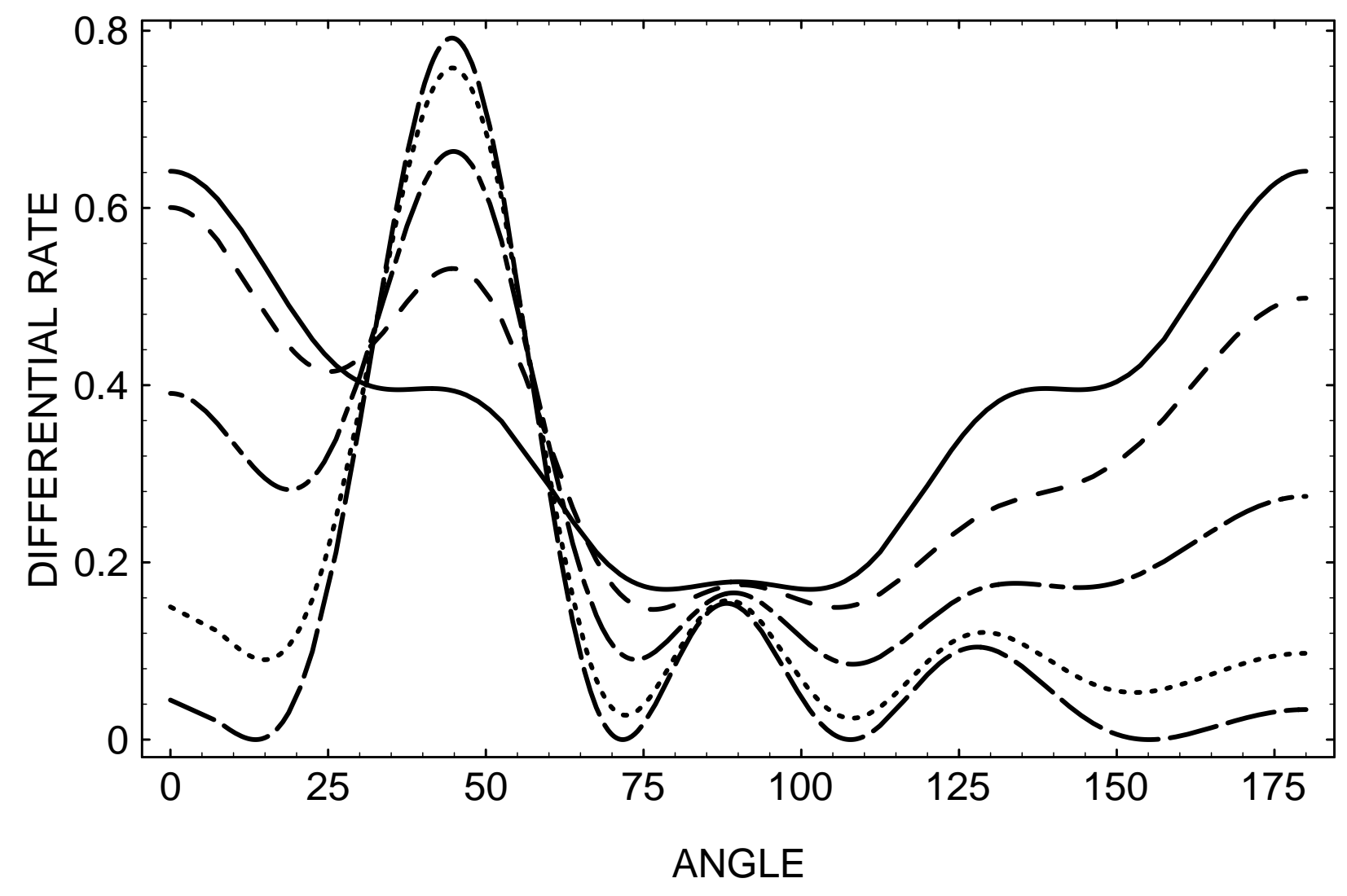




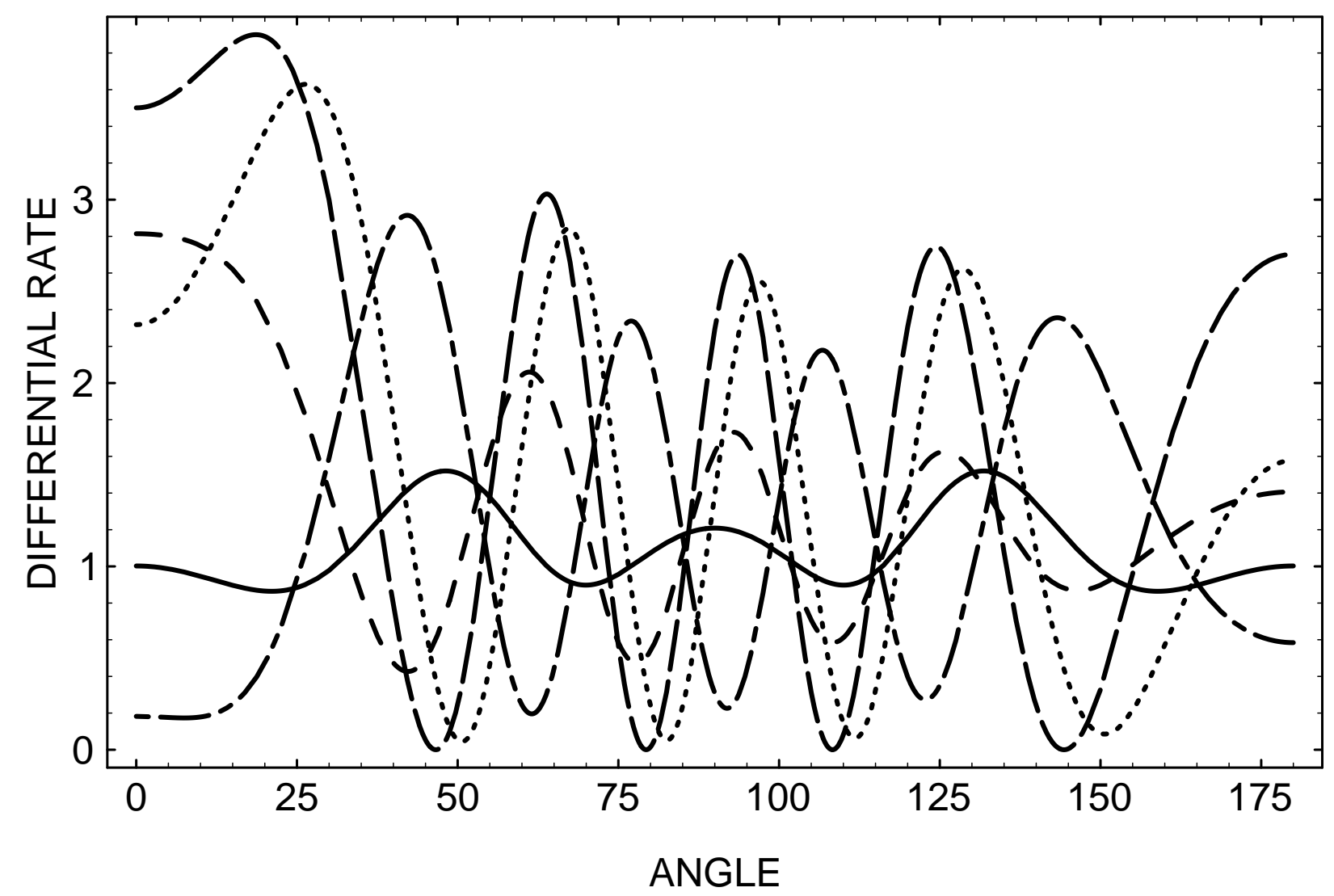




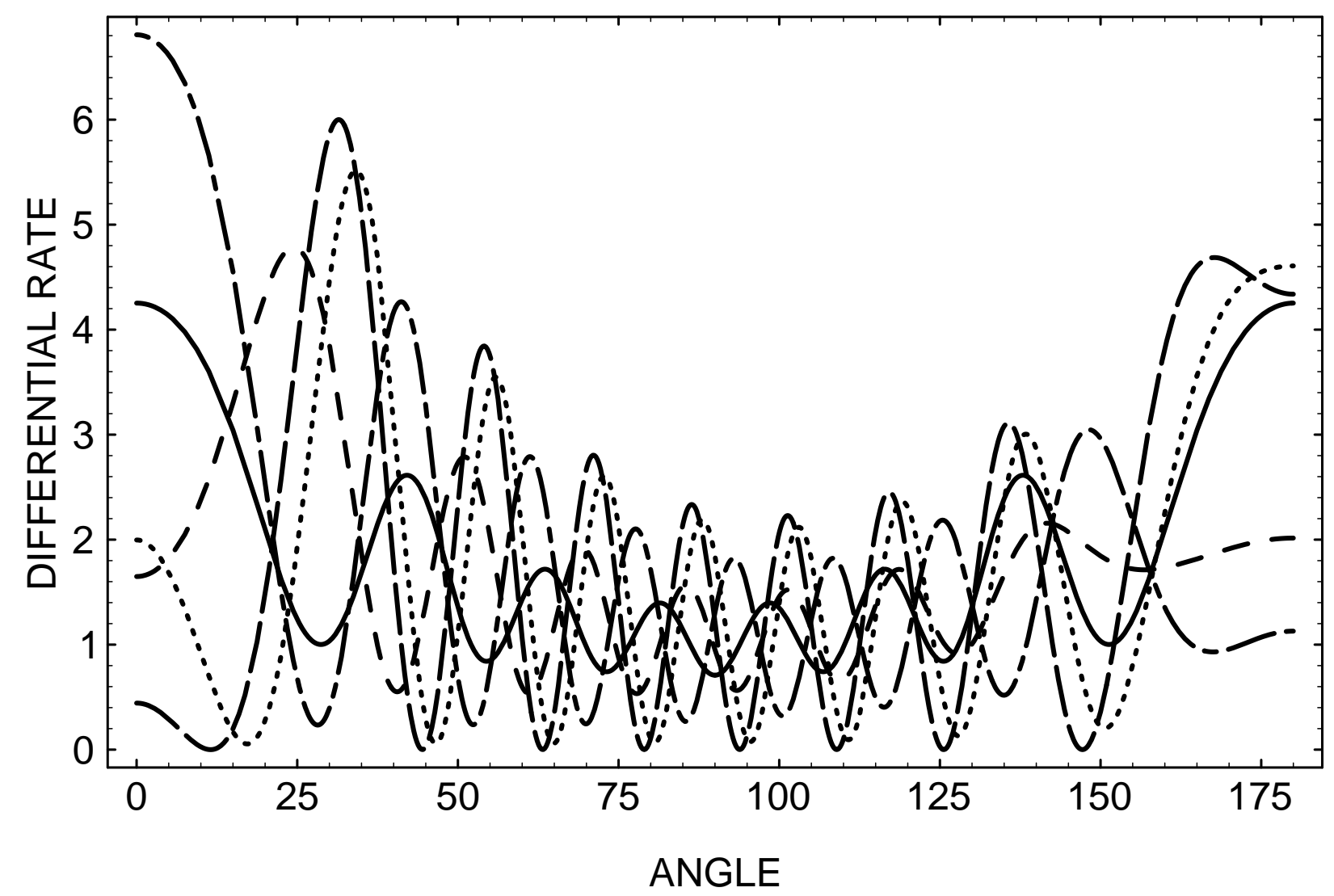




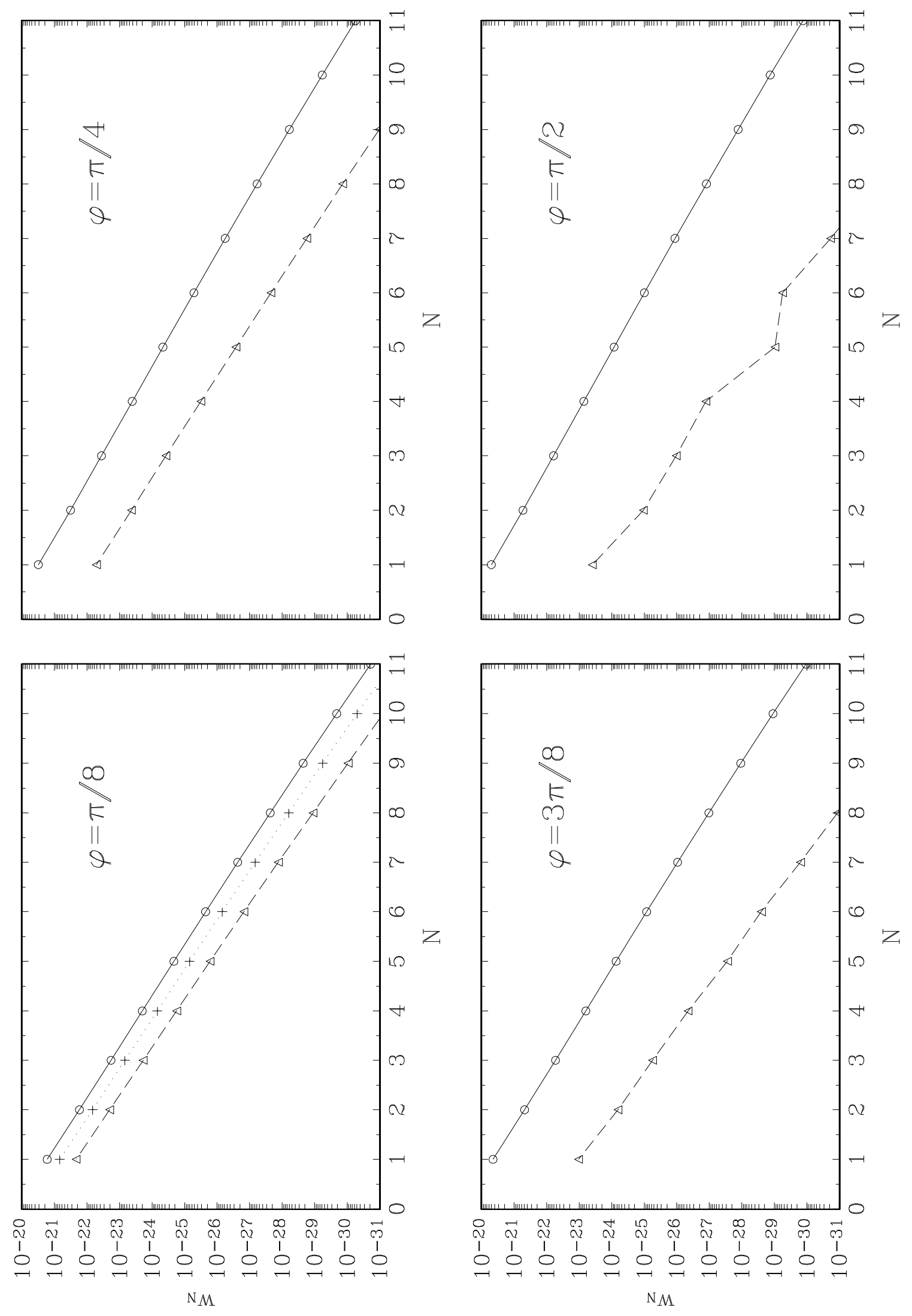




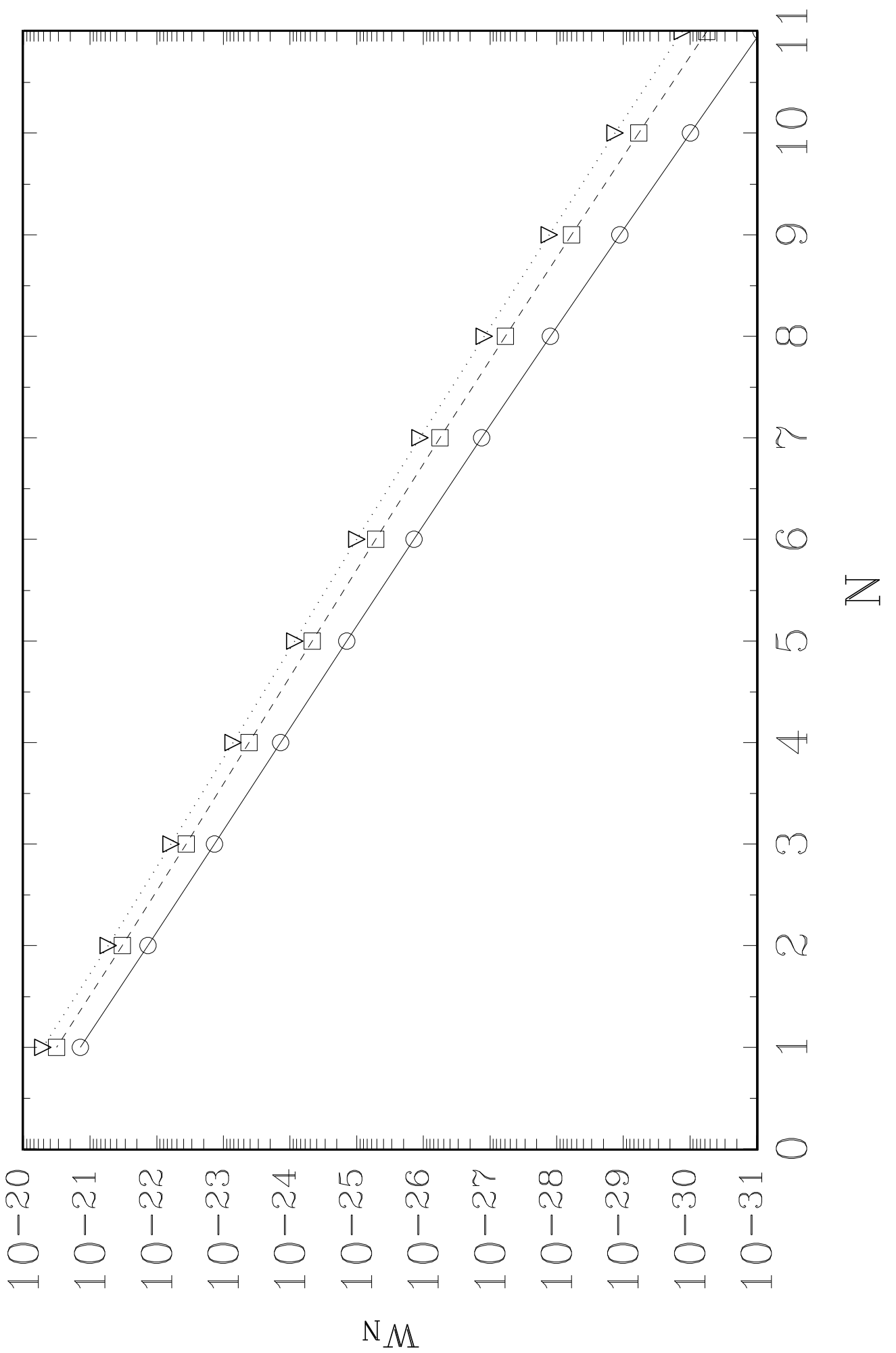



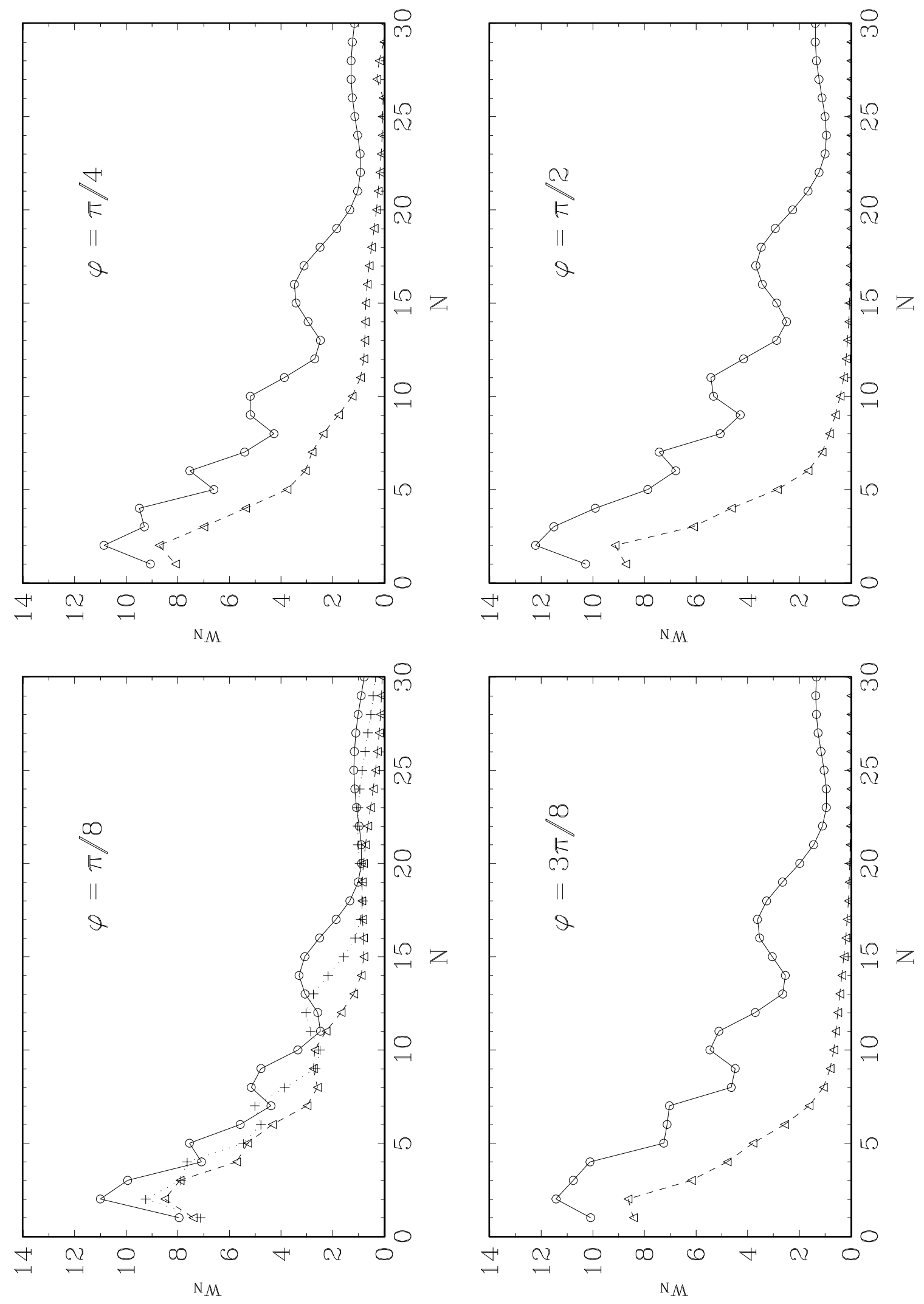


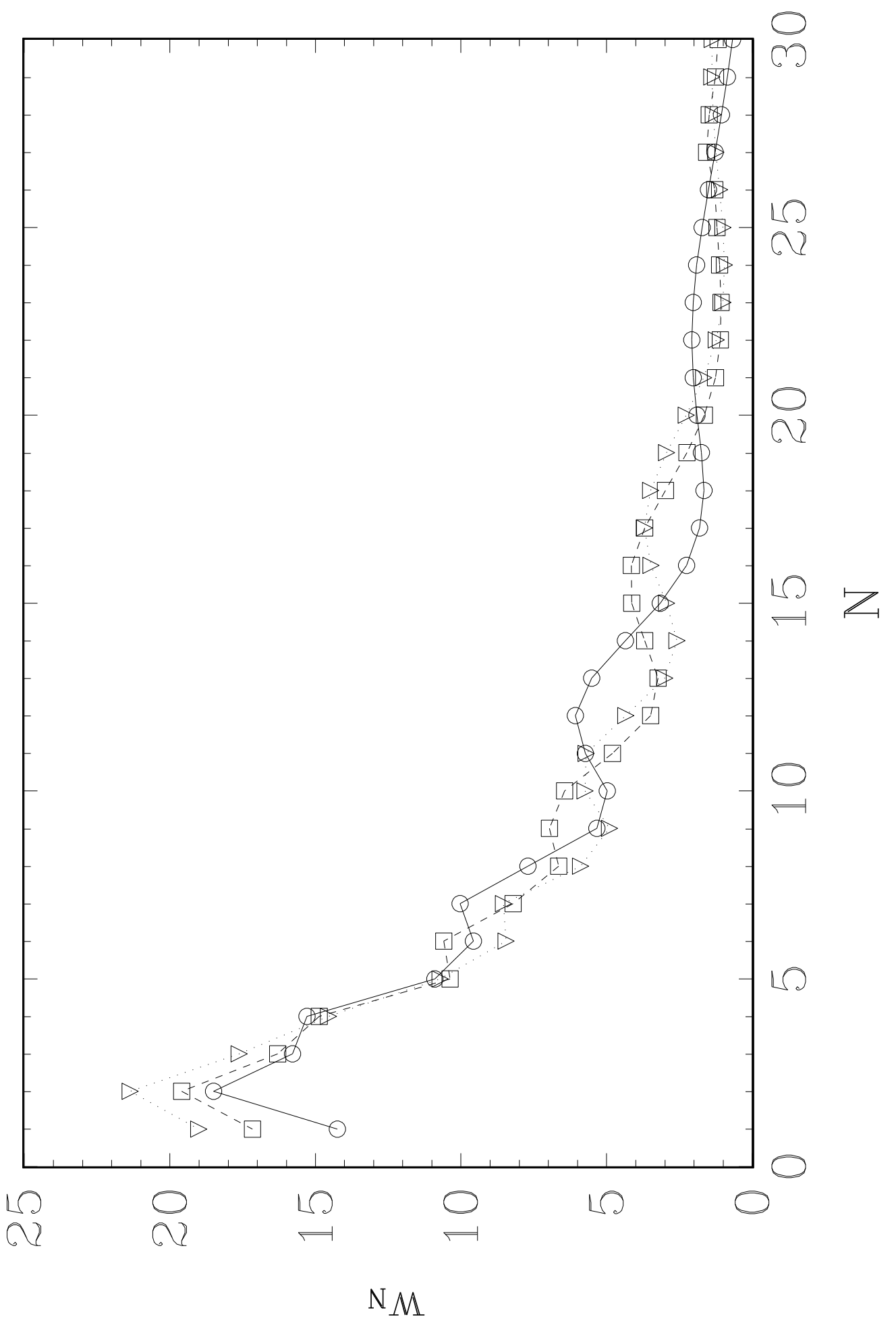

